\title{
Laboratory based X-ray photoemission core-level spectromicroscopy of resistive oxide memories
}

\author{
Daniel M. Gottlob a,*, Eugénie Martinez ${ }^{\mathrm{b}}$, Claire Mathieu ${ }^{\mathrm{a}}$, Christophe Lubin ${ }^{\mathrm{a}}$, \\ Nicolas Chevalier ${ }^{b}$, Munique Kazar Mendes ${ }^{b}$, Christelle Charpin ${ }^{b}$, Eric Jalaguier ${ }^{b}$, \\ Olivier Renault ${ }^{\mathrm{b}}$, Nicholas Barrett ${ }^{\mathrm{a}}$
}

a SPEC, CEA, CNRS, Université Paris Saclay, Gif-sur-Yvette F-91191, France

${ }^{\mathrm{b}}$ Université Grenoble Alpes, F-38000 Grenoble France - CEA, LETI, MINATEC Campus, Grenoble F-38054, France

\section{A R T I C L E I N F O}

\section{Article history:}

Received 30 November 2016

Revised 14 February 2017

Accepted 22 March 2017

Available online xxx

\section{MSC:}

00A79

Keywords:

Resistive switching

$\mathrm{HfO}_{2}$

PEEM

\begin{abstract}
A B S T R A C T
$\mathrm{HfO}_{2}$-based resistive oxide memories are studied by core-level spectromicroscopy using a laboratorybased X-ray photoelectron emission microscope (XPEEM). After forming, the top electrode is thinned to about $1 \mathrm{~nm}$ for the XPEEM analysis, making the buried electrode/ $/ \mathrm{HfO}_{2}$ interface accessible whilst preserving it from contamination. The results are obtained in the true photoemission channel mode from individual memory cells $(5 \times 5 \mu \mathrm{m})$ excited by low-flux laboratory X-rays, in contrast to most studies employing the X-ray absorption channel using potentially harmful bright synchrotron X-rays. Analysis of the local $\mathrm{Hf} 4 \mathrm{f}, \mathrm{O} 1 \mathrm{~s}$ and Ti $2 \mathrm{p}$ core level spectra yields valuable information on the chemistry of the forming process in a single device, and in particular the central role of oxygen vacancies thanks to the spectromicroscopic approach.
\end{abstract}

(c) 2017 Published by Elsevier B.V.

\section{Introduction}

Oxide resistive random access memories (OxRRAMs) are promising candidates in the search for fast, low power memory devices for cache, system and storage applications [1]. Hafnium based OxRRAMs take a special place, because they can be readily integrated into existing semiconductor technology [2]. However, the chemistry of the resistive switching mechanism still needs better understanding to bring the technology to mass production readiness levels [3].

Investigations of the chemistry of the forming and switching processes point to filamentary conduction and cation reduction near the interface with the electrode. While X-ray photoemission spectroscopy (XPS) provides high chemical selectivity, including for buried layers underneath a thin top electrode, the probed area, defined by the beam spot sizes typically in the range of 0.1$1 \mathrm{~mm}$, limits its use for near-technology devices, as filaments are often well below the micrometer scale [4,5]. Furthermore, typical XPS sensitivity is about $1 \%$ making such area-averaged analysis of nanometric filaments challenging. By reducing the analyzed area in photoemission to a single device, the filament to surrounding

\footnotetext{
* Corresponding author.

E-mail addresses: daniel.gottlob@cea.fr, daniel.gottlob@gmail.com (D.M. Gottlob).
}

oxide ratio is significantly enhanced and the chemical sensitivity increased.

Photoelectron emission microscopy with hemispherical energy analyzers provides the simultaneous lateral and energetic resolution necessary to investigate the chemistry of single OxRRAM devices. However, a major obstacle is that the overall transmission of the PEEM optics and energy analyzer is rather low [6]. Furthermore, when using a laboratory X-ray source such as $\mathrm{Al} \mathrm{K} \alpha$, the photoionization cross-section for many core levels is weak compared to that possible when using soft X-ray energy typical of synchrotron light sources. Nevertheless, in this paper we show that a highly stable laboratory XPEEM setup can compensate to some extent by much longer acquisition times than those usually used at synchrotron sources, additionally avoiding any possible sample modification by the use of a low-flux X-ray source.

The availability of such highly stable laboratory based XPEEM is also of considerable interest when allied with complementary characterization techniques such as scanning Auger nanoprobes (SAM), scanning electron microscopy (SEM), conductive atomic force microscopy (C-AFM), $\mathrm{Ar}^{+}$sputtering and gas cluster ion beam milling. The lateral resolution in core level spectroscopic imaging of about $0.5 \mu \mathrm{m}$ [7] is sufficient to select single devices of several $\mu \mathrm{m}$ size, ensuring that measured chemical changes can be directly related to the area in which the switching occurs, rather than to spurious edge effects or changes in background signal inevitably 


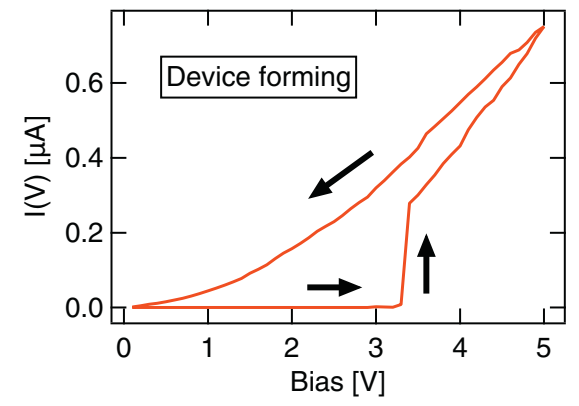

Fig. 1. C-AFM curve of the forming process with $5 \mathrm{~V}$ bias and $1 \mathrm{~mA}$ compliance current.

present in XPS area-averaged over several devices and the intervening oxide regions. Besides, it was shown previously that a high energy resolution can be maintained at the highest level $(<0.5 \mathrm{eV})$ [7] even at a good lateral resolution, which is of prime importance for getting direct chemical fingerprinting from individual devices.

In this paper, we report on threshold, $\mathrm{Hf} 4 \mathrm{f}, \mathrm{O} 1 \mathrm{~s}$ and $\mathrm{Ti} 2 \mathrm{p}$ core-level XPEEM microscpectroscopic analysis of the forming process in individual resistive memory devices, i.e. the changes between the virgin and low resistance state (VS and LRS) of thin film devices of near-technological size.

\section{Experiment}

The samples were made in the Silicon Technology Platform of CEA-LETI (Grenoble, France). The TiN(20 nm)/Ti(10 nm)/ $\mathrm{HfO}_{2}(10 \mathrm{~nm}) / \mathrm{TiN}(40 \mathrm{~nm})$ heterostructure was grown on the $\mathrm{Si}$ substrate by physical vapor (for the TiN and Ti electrode layers) and atomic layer (for the $\mathrm{HfO}_{2}$ resistive oxide) deposition. $(5 \times 5) \mu \mathrm{m}^{2}$ top electrodes were patterned into a grid with $5 \mu \mathrm{m}$ device spacing. The devices were individually electroformed by Conducting tip Atomic Force Microscopy (C-AFM) with a compliance current of $1 \mathrm{~mA}$ (Fig. 1). However, the low resistance state of all devices only registered $800 \mu \mathrm{A}$ at the $5 \mathrm{~V}$ maximum bias. The devices had, on average, a resistance of $R_{V S}=465 \mathrm{M} \Omega$ in the $V S$ and $R_{L R S}=6.5$ $\mathrm{k} \Omega$ in the LRS, resulting in a resistance ratio of about 71,500 . The third, near linear branch is the ramping down of the current with bias in the LRS.

In order to perform XPEEM measurements of the $\mathrm{HfO}_{2}$ layer, we thinned the top electrode after C-AFM forming using first $\mathrm{Ar}^{+}$ sputtering ( $21 \mathrm{~min}$., $\mathrm{Ar}^{+}$ion energy of $2 \mathrm{keV}$, ion current on sample of $2 \mu \mathrm{A}$ in a $4 \mathrm{~mm}^{2}$ target area) and then Gas Cluster Ion Beam (GCIB) milling (10 min at $15 \mathrm{keV}, 13 \mathrm{nA}, 4 \mathrm{~mm}^{2}$ ). Clusters of 2500 $\mathrm{Ar}$ atoms were used, giving an average energy per atom of $6 \mathrm{eV}$ $[8,9]$. This procedure was used to minimize possible cascade damage in the $\mathrm{HfO}_{2}$ which could result from using high energy $\mathrm{Ar}^{+}$ ions to thin the electrode down to $1 \mathrm{~nm}$ [10]. The milling conditions were determined by previous calibration samples. A sketch of the final sample structure before XPEEM analysis is shown in Fig. 2.

Scanning Auger electron spectroscopy in a $25 \mu \mathrm{m}$ field of view using a $30 \mathrm{~nm}$ electron beam and $10 \mathrm{keV}$ primary energy was used to analyze the composition of the sample after all XPEEM experiments had been carried out. An SEM image obtained in the Auger system in Fig. 3 shows the OxRRAM devices on the sample surface. Two areas were analyzed, marked by the blue (on a device) and red (outside the devices) circles. The corresponding Auger spectra are presented in Fig. 4. The lower (red) Auger spectrum shows that the thinning procedure completely removed the exposed $\mathrm{HfO}_{2}$ layer between the devices, leaving only Ti and $\mathrm{N}$ of the bottom electrode in the Auger spectrum. The signal from the devices shows a contribution of $\mathrm{Hf}$ and $\mathrm{O}$ Auger electrons from the

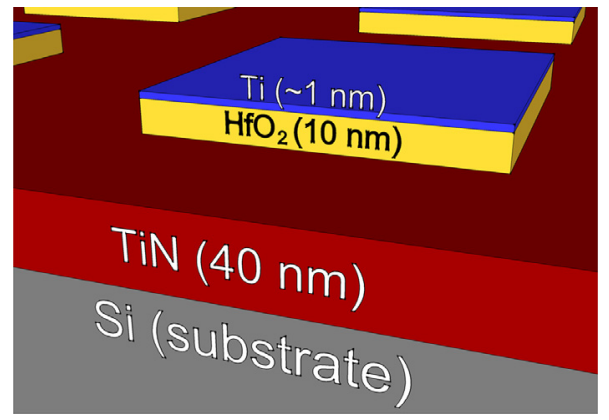

Fig. 2. Sketch of sample geometry after thinning procedure.

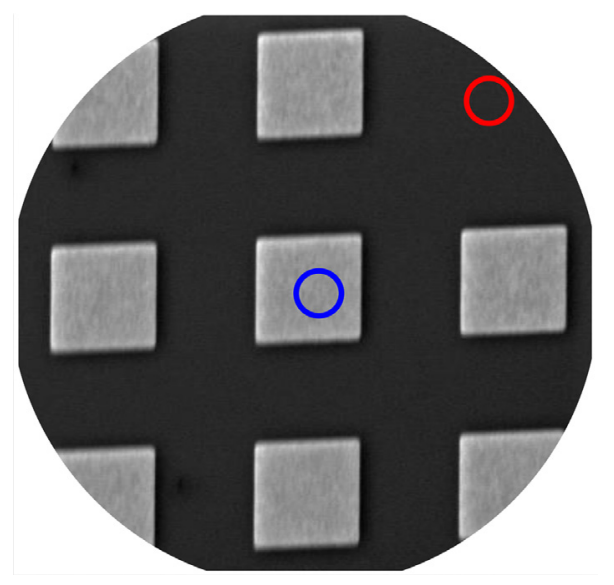

Fig. 3. SEM image showing nano-Auger measuring spots as blue and red circles. The measurement areas were $30 \mathrm{~nm}$ in the center of the circles; devices are $(5 \times$ 5) $\mu^{2}$. (For interpretation of the references to color in this figure legend, the reader is referred to the web version of this article.)

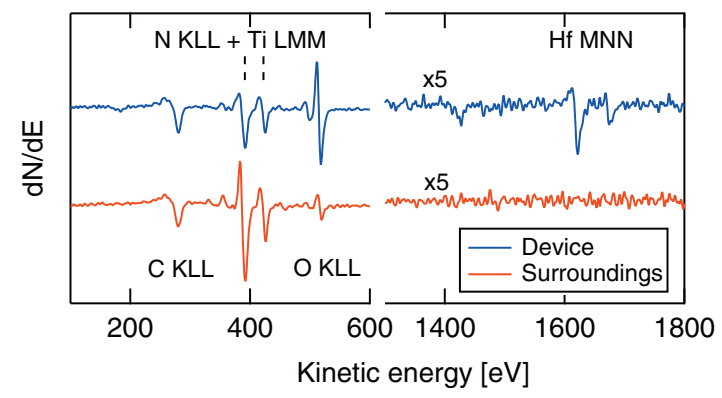

Fig. 4. Nano-Auger spectra after thinning of the top electrodes from the devices and surrounding material and XPEEM experiments.

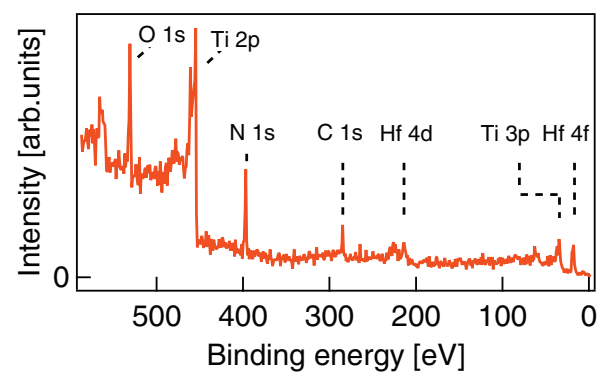

Fig. 5. XPS survey scan after transfer to the XPEEM.

resistive oxide, in addition to the Ti Auger electrons from the $1 \mathrm{~nm}$ top electrode. In the latter case, the signature of the combined $\mathrm{Ti}$ and $\mathrm{N}$ peaks confirm that the Ti signal comes from the residual TiN top electrode whereas between devices it is due to the exposed 
a)

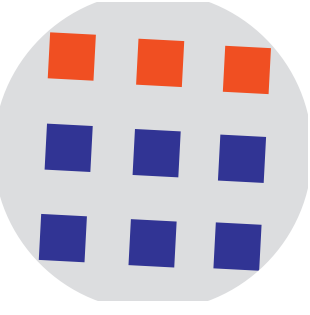

b)

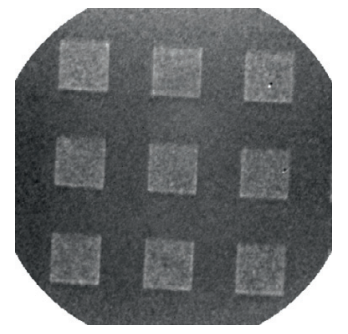

c)

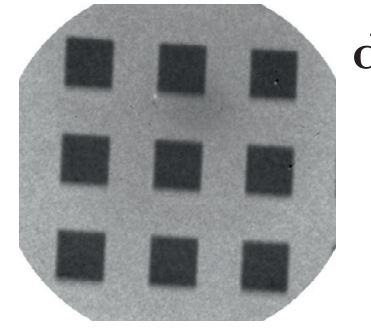

d)

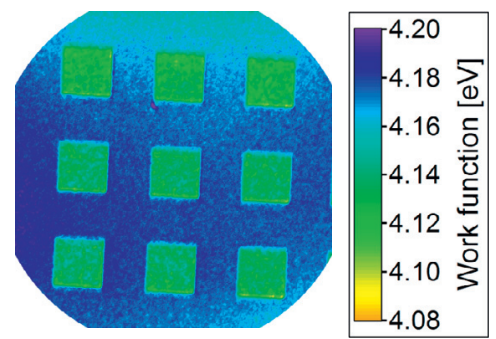

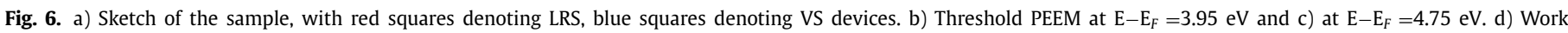
function map. Devices are $(5 \times 5) \mu \mathrm{m}^{2}$. (For interpretation of the references to color in this figure legend, the reader is referred to the web version of this article.)

Table 1

Mesurement acquisition details.

\begin{tabular}{llll}
\hline Transition & Hf $4 \mathrm{f}$ & O $1 \mathrm{~s}$ & Ti 2p \\
\hline BE Range [eV] & $12-22$ & $528.2-534.7$ & $452.2-464.7$ \\
$\Delta \mathrm{E}[\mathrm{eV}]$ & 0.1 & 0.1 & 0.1 \\
Exposure [s] & 60 & 60 & 60 \\
Sweeps & 45 & 15 & 60 \\
Time [h] & 61 & 16.5 & 31.5 \\
\hline
\end{tabular}

bottom electrode. Carbon peaks are present in both spectra due to surface contamination. We assume that this does not influence the chemistry of the forming process.

The XPS survey spectrum obtained in the XPEEM microscope directly after sputtering and transfer using a nitrogen filled capsule to the XPEEM system is shown in Fig. 5. The Ti, N, Hf and O corelevel lines can be identified, as well as $C$.

The XPEEM is a NanoESCA Mk1 (ScientaOmicron) type. The microscope comprises a fully electrostatic PEEM column and an imaging double energy analyzer [11]. All measurements were made by illuminating the sample with a high intensity, monochromatic Al $\mathrm{K} \alpha$ X-ray source. The overall energy resolution (PEEM plus X-ray linewidth) was $0.49 \mathrm{eV}$. The $\mathrm{O} 1 \mathrm{~s}$, Ti $2 \mathrm{p}$, and $\mathrm{Hf} 4 \mathrm{f}$ electrons have inelastic mean free paths in the Ti electrode material of $2.56,2.73$ and $3.57 \mathrm{~nm}$, respectively [12].

Fig. 6a shows a sketch of the devices analyzed by XPEEM. Both virgin state (blue, lower six) and formed (red, upper three) devices are present. Fig. $6 \mathrm{~b}$ and $\mathrm{c}$ show two typical images taken from a photoemission threshold image series. The electron energies are 3.95 and $4.75 \mathrm{eV}$, respectively, measured with respect to the sample holder Fermi level $\left(E-E_{F}\right)$. The contrast inversion between Fig. $6 \mathrm{~b}$ and $\mathrm{c}$ is due to the work function difference between Ti top and TiN bottom electrodes. Fig. 6d shows a work function map, obtained from a pixel-by-pixel fit of the threshold spectra using an error function. The devices and their surroundings clearly display two different work functions. The Ti top and TiN bottom electrodes work functions are $(4.13 \pm 0.005)$ and $(4.17 \pm 0.005)$ $\mathrm{eV}$, respectively, in agreement with previous literature [13].

We then analyzed the same devices using core level, laboratory based spectroscopic XPEEM. Three image series were acquired with a combined acquisition time of 109 hours, as displayed in Table 1.

\section{Results and discussion}

Fig. 6a shows a sketch of the XPEEM field of view, identifying the devices switched to the LRS in red and VS devices in blue. In order to better visualize the spatial variations of the chemical states, Fig. 7a-d show the sum of images taken over binding energy ranges of $16.4-19.9 \mathrm{eV}$ for $\mathrm{Hf} 4 \mathrm{f}, 529.7-531.8 \mathrm{eV}$ for $\mathrm{O} 1 \mathrm{~s}$, and 453.9-458 eV and 458.6-460 eV for the two Ti $2 \mathrm{p}$ contributions of $\mathrm{TiN}$ and $\mathrm{TiO}_{2}$. This is equivalent to integrating the image intensities over the main core level peak as shown in Fig. 8. Hf and $\mathrm{O}$ intensities are maximum in the devices (Fig. 7a and b). The signal from the Ti top electrode is filtered out of Fig. 7c by the energy range of the image integration, which corresponds to TiN. The integration over the $\mathrm{Ti} 2 \mathrm{p}$ peak range for $\mathrm{TiO}_{2}$ results in Fig. $7 \mathrm{~d}$. The contrast inversion with respect to Fig. $7 \mathrm{c}$ shows that the Ti on the electrodes is oxidized, while the surrounding material is TiN.

Local spectra were extracted from the image series by selecting regions of interest (ROIs) on the VS and LRS state devices and between them. The local $\mathrm{Hf} 4 \mathrm{f}, \mathrm{O} 1 \mathrm{~s}$ and $\mathrm{Ti} 2 \mathrm{p}$ spectra are shown in Fig. 8a-d. The LRS Hf $4 \mathrm{f}$ spectrum is shifted by $0.09 \mathrm{eV}$ to higher binding energy compared with the VS spectrum, confirmed by the double peak structure in the difference spectrum. This shift may be compared with the $0.18 \mathrm{eV}$ shift reported previously [14], attributed to n-type doping by the oxygen vacancies created in the switching process. Note that the latter measurements were done with a much higher compliance current $(10 \mathrm{~mA})$ which probably yields to a higher defect concentration and a wider conduction path. a)

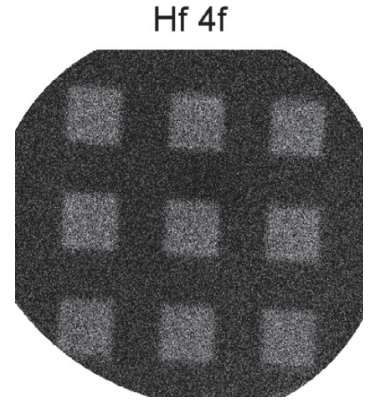

b)

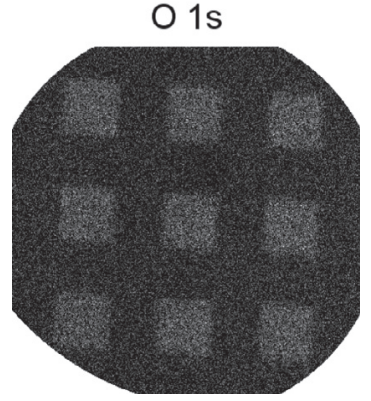

c)

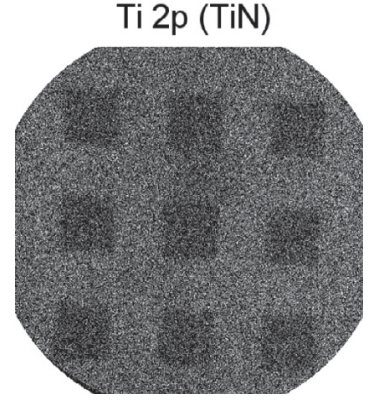

d)

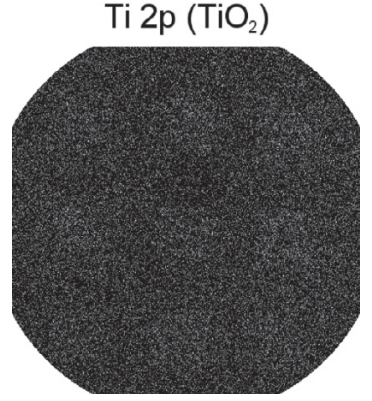

Fig. 7. a-d) Integrated image intensities of Hf $4 \mathrm{f}(16.4-19.9 \mathrm{eV}), \mathrm{O} 1 \mathrm{~s}(529.7-531.8 \mathrm{eV})$ and Ti $2 \mathrm{p}(453.9-458 \mathrm{eV}$ and $458.6-460 \mathrm{eV})$. Devices are $(5 \times 5) \mu \mathrm{m}^{2}$. 
a)

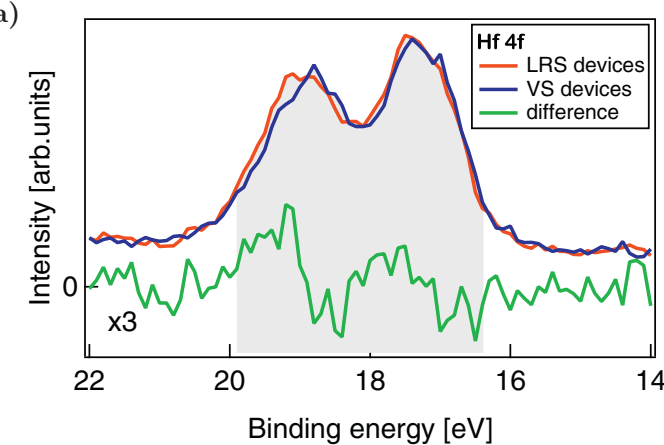

b)

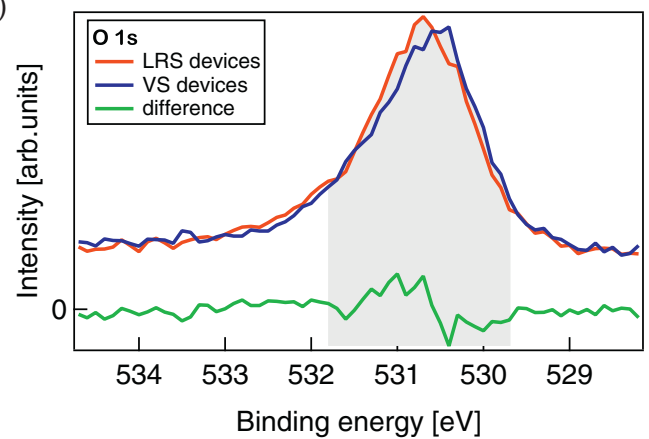

c)

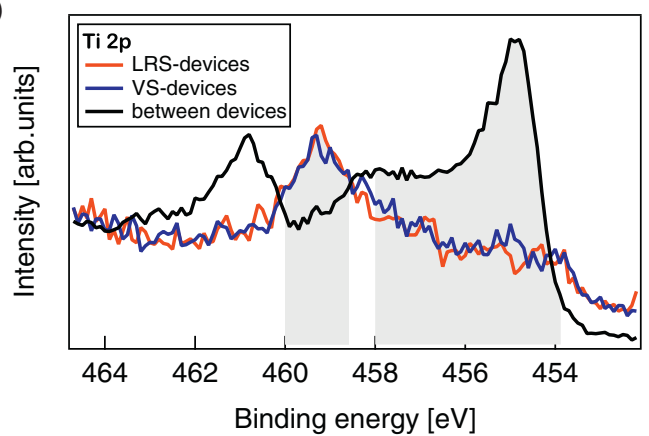

The same core level shift is observed on both $\mathrm{Hf} 4 \mathrm{f}$ and $\mathrm{O} 1 \mathrm{~s}$ core level spectra. As already reported in the literature [14], this is thought to be related to a Fermi level pinning toward the conduction band due to n-type defects such as electrons trapped at oxygen vacancy sites. The core-level shift from this study is in agreement with these observations and thus underlines the key role of oxygen vacancies in the resistive switching mechanism. In the model that has been associated with the observation in the literature, Oxygen anions $\left(\mathrm{O}_{2}^{-}\right)$are injected and trapped in the Ti getter layer when positively biasing the TiN/Ti top electrode, thus leading to a conducting path inside the active $\mathrm{HfO}_{2}$ layer.

Such vacancies are unstable and thus particularly difficult to highlight by physicochemical characterization. Literature results are mainly based on synchrotron based X-ray photoelectron spectroscopy $[14,16]$ to overcome the rather low sensitivity of laboratory measurements. We show here that this can be also done by XPEEM measurements thanks to higher spatial resolution, provided that the low sampling depth is circumvented by top electrode removal before analysis. Advanced microspectroscopy can thus provide direct evidence of the chemical processes involved in the resistive switching mechanism.

\section{Conclusion}

$\mathrm{HfO}_{2}$ based OxRRAM devices, switched from the VS to the LRS were investigated by laboratory-based core-level XPEEM. The measurements of buried $\mathrm{HfO}_{2}$ layers were performed with a monochromatized $\mathrm{Al} \mathrm{K} \alpha \mathrm{X}$-ray source, while the high stability of the XPEEM experiment allowed for exposure times in the day-scale to equilibrate the rather low transmission of the overall system. The spectra showed a rigid shift of $0.09 \mathrm{eV}$ to higher BE for LRS Hf $4 \mathrm{f}$ and $\mathrm{O} 1 \mathrm{~s}$ compared to the virgin state. The spatially resolved $\mathrm{Ti}$ $2 \mathrm{p}$ core level spectra also confirm the role of the Ti top electrode as an oxygen reservoir/getter.

\section{Acknowledgments}

This work has been partially supported by the European 621217 PANACHE project and by the CEA Operando Project. The work was performed at the Platform For Nano-Characterization (PFNC) of CEA-MINATEC

\section{References}

The LRS 0 1s spectrum in Fig. $8 \mathrm{~b}$ also shows a shift to higher binding energy and a slight change peak shape, which again can be seen in the difference spectrum. The LRS spectrum is shifted by $0.09 \mathrm{eV}$ to higher binding energies compared to the VS spectrum. The $\mathrm{O} 1 \mathrm{~s}$ binding energies in both $\mathrm{TiO}$ (530 eV [15]) and $\mathrm{HfO}_{2}$ (531 eV [14]) are well within the range of our recorded $\mathrm{O} 1 \mathrm{~s}$ peak. A change in relative intensity due to the migration of oxygen atoms from the Hf to the Ti during forming could account for the change in peak shape, whereas the n-type doping could account for the shift in binding energy.

The Ti 2p spectra (Fig. 8c) recorded between the devices and on the devices are quite different. The peak binding energies of approx. $454.9 \mathrm{eV}$ (between devices) and $459.2 \mathrm{eV}$ (on devices) agree with literature values of $\mathrm{TiN}$ and $\mathrm{TiO}_{2}$ [15]. This reflects the distinct chemical composition of two different Ti layers as shown in the characterization. The signal between the devices originates from the TiN bottom electrode revealed by the milling treatment, while the signal recorded from the devices represents the residual $\mathrm{Ti} / \mathrm{TiO}_{x}$ top electrode (see Fig. 2). The presence of oxidized $\mathrm{Ti}$ confirms the role of $\mathrm{Ti}$ as an oxygen getter during the resistive switching process. However, no significant difference between the LRS and VS can be distinguished in the Ti $2 p$ spectra.

[1] B. Govoreanu, G.S. Kar, Y.Y. Chen, V. Paraschiv, S. Kubicek, a. Fantini, I.P. Radu, L. Goux, S. Clima, R. Degraeve, N. Jossart, O. Richard, T. Vandeweyer, K. Seo P. Hendrickx, G. Pourtois, H. Bender, L. Altimime, D.J. Wouters, J.a. Kittl, M. Jurczak, $1010 \mathrm{Nm} 2 \mathrm{HF} / \mathrm{HfO} \mathrm{x}$ crossbar resistive RAM with excellent performance, reliability and low-energy operation, in: Technical Digest - International Electron Devices Meeting, IEDM, 2011, pp. 729-732, doi:10.1109/IEDM. 2011.6131652.

[2] P. Calka, E. Martinez, V. Delaye, D. Lafond, G. Audoit, D. Mariolle, N. Chevalier, H. Grampeix, C. Cagli, V. Jousseaume, C. Guedj, Chemical and structural properties of conducting nanofilaments in TiN/HfO2-based resistive switching structures., Nanotechnology 24 (8) (2013) 085706, doi:10.1088/0957-4484/24/ $8 / 085706$.

[3] B. Traor, P. Blaise, E. Vianello, H. Grampeix, S. Jeannot, L. Perniola, B. De Salvo, Y. Nishi, On the origin of low-resistance state retention failure in HfO2-based RRAM and impact of doping/alloying, IEEE Trans. Electron. Devices 62 (12) (2015) 4029-4036, doi:10.1109/TED.2015.2490545.

[4] C. Baeumer, C. Schmitz, A. Marchewka, D.N. Mueller, R. Valenta, J. Hackl, N. Raab, S.P. Rogers, M.I. Khan, S. Nemsak, M. Shim, S. Menzel, C.M. Schneider, R. Waser, R. Dittmann, Quantifying redox-induced schottky barrier variations in memristive devices via in operando spectromicroscopy with graphene electrodes, Nat. Commun. 7 (May) (2016) 12398, doi:10.1038/ncomms12398.

[5] R. Waser, M. Aono, Nanoionics-based resistive switching memories., Nat. Mater 6 (11) (2007) 833-840, doi:10.1038/nmat2023.

[6] M. Escher, K. Winkler, O. Renault, N. Barrett, Applications of high lateral and energy resolution imaging XPS with a double hemispherical analyser based spectromicroscope, J. Electron. Spectros. Relat. Phenomena. 178-179 (C) (2010) 303-316, doi:10.1016/j.elspec.2009.06.001. 
[7] O. Renault, M. Lavayssire, A. Bailly, D. Mariolle, N. Barrett, Core level photoelectron spectromicroscopy with al $\mathrm{k} 1$ excitation at $500 \mathrm{~nm}$ spatial resolution, J. Electron. Spectros. Relat. Phenomena. 171 (13) (2009) 68-71. http://dx.doi. org/10.1016/j.elspec.2009.03.008. http://www.sciencedirect.com/science/article/ pii/S0368204809000711

[8] R. Grilli, R. Simpson, C.F. Mallinson, M.A. Baker, Comparison of ar+ monoatomic and cluster ion sputtering of ta205 at different ion energies, by XPS: part 1 - monoatomic ions, Surf. Sci. Spectra 21 (1) (2014a) 50-67. http://dx. doi.org/10.1116/11.20140701. http://scitation.aip.org/content/avs/journal/sss/21/ 1/10.1116/11.20140701

[9] R. Grilli, R. Simpson, C.F. Mallinson, M.A. Baker, Comparison of ar+ monoatomic and cluster ion sputtering of ta2o5 at different ion energies, by XPS: part 2 cluster ions, Surf. Sci. Spectra 21 (1) (2014b) 68-83, doi:10.1116/11.20140702.

[10] A. Delcorte, M. Debongnie, Macromolecular sample sputtering by large Ar and CH4 clusters: elucidating chain size and projectile effects with molecular dynamics, J. Phys. Chem. C 119 (46) (2015) 25868-25879, doi:10.1021/acs.jpcc. 5 b07007.

[11] M. Escher, N. Weber, M. Merkel, B. Krmker, D. Funnemann, S. Schmidt, F. Reinert, F. Forster, S. Hfner, P. Bernhard, C. Ziethen, H.J. Elmers, G. Schnhense, Nanoesca: imaging \{UPS $\}$ and $\{$ XPS $\}$ with high energy resolution, J. Electron. Spectros. Relat. Phenomena 144147 (2005) 1179-1182 Proceeding of the Fourteenth International Conference on Vacuum Ultraviolet Radiation Physics, http: //dx.doi.org/10.1016/j.elspec.2005.01.250.
[12] W.H. Gries, A universal predictive equation for the inelastic mean free pathlengths of X-ray photoelectrons and auger electrons, Surf. Interface Anal. 24 (1) (1996) 38-50, doi:10.1002/(SICI)1096-9918(199601)24:1〈38::AID-SIA84〉3.0.CO; 2-H.

[13] F. Fillot, T. Morel, S. Minoret, I. Matko, S. Matrejean, B. Guillaumot, B. Chenevier, T. Billon, Investigations of titanium nitride as metal gate material, elaborated by metal organic atomic layer deposition using \{TDMAT\} and \{NH3\}, Microelectron. Eng. 82 (34) (2005) 248-253. Proceedings of the ninth european workshop on materials for advanced metallization 2005, http://dx. doi.org/10.1016/j.mee.2005.07.083

[14] T. Bertaud, D. Walczyk, C. Walczyk, S. Kubotsch, M. Sowinska, T. Schroeder, C. Wenger, C. Valle, P. Gonon, C. Mannequin, V. Jousseaume, H. Grampeix, Resistive switching of HfO2-based metalinsulatormetal diodes: impact of the top electrode material, Thin Solid Films 520 (14) (2012) 4551-4555. Proceedings of the \{EMRS 2011 Spring Meeting Symposium D: Synthesis, Processing and Characterization of Nanoscale Multi Functional Oxide Films \{III\}, http:// dx.doi.org/10.1016/j.tsf.2011.10.183

[15] N.C. Saha, H.G. Tompkins, Titanium nitride oxidation chemistry: an xray photoelectron spectroscopy study, J. Appl. Phys. 72 (7) (1992) 3072-3079. http: //dx.doi.org/10.1063/1.351465.

[16] P. Calka, E. Martinez, D. Lafond, S. Minoret, S. Tirano, B. Detlefs, J. Roy, J. Zegenhagen, C. Guedj, Origin of resistivity change in NiO thin films studied by hard X-ray photoelectron spectroscopy, J. Appl. Phys. 109 (12) (2011) 10-16, doi:10.1063/1.3596809. 